\title{
Ubiquitous Mobile Real Time Visual Translator Using Augmented Reality for Bahasa Language
}

\author{
Behrang Parhizkar, Keamogetswe Oteng, One Ndaba, Arash Habibi Lashkari, and Zahra Mohana Gebril
}

\begin{abstract}
Mobile technology have rapidly advanced over the years, successful researches of augmented reality on mobile phones under the different mobile platforms have made it possible to implement augmented reality technology on mobiles. Now the possibility of combining real time visual with augmented reality seems to be a success. This paper is simply to emphasize on combining real time visual with augmented reality technology on mobiles for education purpose. The research is based on how the implementation of real time visual translator on augmented reality mobile applications can help people in understanding/translating and give meanings to some writings displayed around them ubiquitously.
\end{abstract}

Index Terms-Education for undergraduates, mobile augmented reality, mobile applications, real time, visual and ubiquitous.

\section{INTRODUCTION}

Creating artificial world that provide sense of the real world where a real life is enhanced by virtual elements in real time. Augmented Reality (AR) is "an environment that includes both virtual reality and real-world elements" [1]-[3]. In short augmented reality (AR) is whereby an existing object is taken and information is blended on it. There different forms to provide feedback about Augmented Reality (AR) such as vision, audio, tactile, and other forms. The rapid growth of use of Augmented Reality (AR) is increasing at higher rates. The first augmented reality system was created in 1968 by Ivan Sutherland, which is also the first virtual reality system. About 2 decades later the computer which used clamshell design was released followed by 1992 Smartphone which acted as phone, pager, calculator, address book, fax machine, and e-mail device. Up until today the implementation of Augmented Reality is invested in everyday life. Today Augmented Reality is used in entertainment, military training, engineering design, robotics, manufacturing and other industries [2], [3].

As computers increase in power and decrease in size, new mobile, wearable, and general computing applications are rapidly becoming feasible, providing people access to online resources always and everywhere. This new flexibility makes

Manuscript received November 1, 2012; revised January 4, 2013

Behrang Parhizkar and Arash Habibi Lashkari are with Postgraduate Centre of Study (PGC), Limkokwing University of Creative Technology, Cyberjaya, Malaysia (email: hani.pk@limkokwing.edu.my, A_habibi_L@hotmail.com).

Keamogetswe Oteng, One Ndaba, and Zahra Mohana Gebril are with Faculty of Information and Communication Technology, Limkokwing University of Creative Technology, Cyberjaya, Malaysia (email: rasgudookmg@yahoo.com, zahra.gebril@limkokwing.edu.my). possible new kind of applications that manipulate the person's surrounding perspective. AR systems combine virtual information into a person's physical environment so that he or she will observe that information as existing in their surroundings. Mobile augmented reality systems (MARS) provide this service without limiting the individual's location to a specially equipped area.

Augmented Reality (AR) is currently being used in disciplines of computer science (visualization, robotics, nanotechnology), engineering, applied psychology, education, environmental design, medicine, engineering, tourism, and archaeology. The use of mobile phone is the perfect liberation medium for learning in near future. The concept of augmented reality seems to be succefully implemented on mobiles and by combining Mobile Augmented reality, real time rendering and ubiquitous; the idea is to develop a real time translator that will translate Bahasa language to English language and give meanings of the words translated, anywhere to any environment whenever desired (ubiquitous). This application is mostly based on education that is providing or diverting knowledge to people who actually need it [4], [5].

\section{INITIAL ANALYSIS}

Initial analysis was carried out to describe work performed at many different sites and explains the issues and problems encountered when building Augmented Reality systems. AR applications require giving the user the ability to walk around large environments, even outdoors. This requires making the equipment self-contained and portable.

The result of the study is that one of the most basic problems currently limiting Augmented Reality applications is the registration problem. The objects in the real and virtual worlds must be properly aligned with respect to each other, or the illusion that the two worlds coexist will be compromised More seriously, many applications demand accurate registration [6], [7]. For example, if a user wants translate a worn out word in any place it is going to be difficult for the user because the results will not be accurate because the proper registration of the word would not have been done correctly, Augmented Reality will not be accepted in many applications.

Moving to the other places for further education is over whelming for most of the people because they get to experience other people's cultures. However it is a downfall to be in a foreign country only not to understand anything that goes around you every second of the day. Over the recent years we have observed that there is a language barrier for the students coming in to Malaysia to study. It has proven to be 
difficult for them to get the precise meaning of words because most of the words in work areas and public places are written in Bahasa language. Due to the problem mentioned above it is our responsibility to solve the language barrier for the international students. We have decided to develop the ubiquitous real time visual translator using augmented reality for Bahasa language.

\section{A. Problem Analysis}

Based on the observation and the study of the previous work of it is reasonable and conclusive to say that registration problem has proven to be one of the limitations of augmented reality applications. Translation through augmented reality application must be accurate and more precise for the users. The fact that being unable to understand what other people are saying is mentally stressing, therefore causing a language barrier between local people and international people that is, the people from other countries. This of course limits communication, sharing of ideas between people with different point of views and ideas.

Therefore based on the limitation mentioned above there is a need to design an application that can tackle the language barrier that is been observed and do research more on a better way to improve the translation of worn out word to word using augmented reality. The solution to the problem is to develop an application which will give the user possible translated words if the word to be translated is worn out in any possible way.

Word Lens is a current augmented reality application for the iPhone and iPod Touch (with video camera) which offers real time translation of text. You simply point your device's video camera at a sign and the program translates and superimposes the translated text onto the video in real time [6]-[9].

\section{B. Methods (Solutions)}

Android is a flexible software platform designed to deliver a personalized and customizable user experience on mobile devices. Compared to many existing platforms, Android is truly open to continued innovation and new experiences. The Android is a software stack for mobile devices that includes an operating system, middleware and key applications. The Android SDK provides the tools and APIs necessary to begin developing applications on the Android platform using the Java programming language. Some of its features include the application framework enabling reuse and replacement of components, it also hardware dependent such as Camera, GPS, compass, and accelerometer. Android will ship with a set of core applications including an email client, SMS program, calendar, maps, browser, contacts, and others. All applications are written using the Java programming language. The application is mainly developed to be used by anyone in Malaysia especially those who do not know Bahasa language. It will help them translate the words in Bahasa language to English.

\section{LITERATURE REVIEW}

\section{A. Mobile Learning}

The use of mobile phone is the perfect liberation medium for learning in near future. By adding of augmented reality to mobile phones to generate the artificial world for a person to experience by the sense of vision making learning a lot more fun and interesting. The fact that if you can see something that you do not understand or just willing to learn something that is written in a language that you do not understand, it is emotionally draining. This report is basically based on the application that is to be introduced which will be a mobile application that intends to provide wealthier experiences by overlying words or virtual objects over the scene observed through a camera. The mobile application is a real time visual translator for Bahasa language to English that will be embedded on mobile phones [10]-[12].

\section{B. Augmented Reality}

The rapid growth of use of Augmented Reality (AR) is increasing at higher rates. Creating artificial world that provide sense of the real world where a real life is enhanced by virtual elements in real time.

Augmented reality (AR) can be defined as referring to cases in which a real environment is "augmented" by means of virtual objects (Milgram \& Kishino, 1994). Augmented Reality (AR) is the integration of digital information with live video or the user's environment in real time. Ronald Azuma, Research Leader at Nokia Research Centre states that, Augmented Reality (AR) is "an environment that includes both virtual reality and real-world elements. The first augmented reality system was created in 1968 by Ivan Sutherland, which is also the first virtual reality system.

\section{Mobile Augmented Reality}

AR systems integrate virtual information into a person's physical environment so that he or she will perceive that information as existing in their surroundings. Mobile augmented reality systems (MARS) provide this service without constraining the individual's whereabouts to a specially equipped area.

The idea of AR is related to the concept of virtual reality (VR). VR attempts to create an artificial world that a person can experience and explore interactively, predominantly through his or her sense of vision, but also via audio, tactile, and other forms of feedback. AR also brings about an interactive experience, but aims to supplement the real world, rather than creating an entirely artificial environment around the user.

We define an AR system as one that combines real and computer-generated information in a real environment, interactively and in real time, and that aligns virtual objects with physical ones. AR is a subfield of the broader concept of mixed reality (MR), which further includes simulations predominantly taking place in the virtual domain and not in the real world [13], [14].

In the AR entertainment applications, virtual objects (world) generated with Computer Graphics are overlaid onto the real world. This means that the real 3D world is captured by a camera, and then the virtual objects are superimposed onto the captured images. By seeing the real world through some sort of displays, the users find that the virtual world is mixed with the real world. In such AR applications, the users carry a camera and move around the real world in order to change their view points. Therefore the pose and the position of the moving user's camera should be obtained so that the 
virtual objects can be overlaid at correct position in the real world according to the camera motion. Such camera tracking should also be performed in real-time for interactive operations of the AR applications [15], [16].

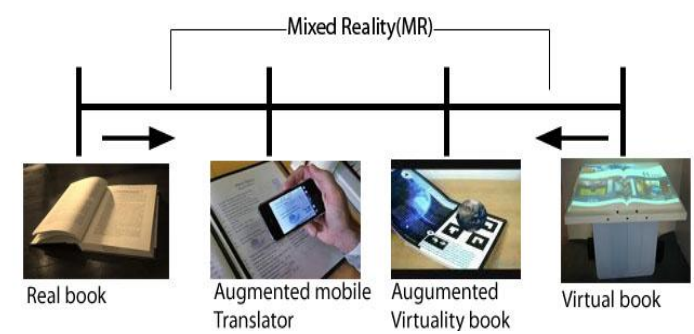

Fig. 1. The physical continuum of realitysource: (Milgram 1998).

Above diagram (Fig. 1) is the physical Continuum of Reality that illustrates the flow of proposed application which was done in relation to the Continuum of Reality by Milgram in 1998 (Fig. 1). Real environment (RE) and virtual environment (VE) are at two sides, mixed reality (MR) is in the middle, AR is near to the real environment side. Data created by the computer can augment real environment and enhance user's comprehension about environment. Augmented Virtually (AV) is a term created by Milgram. It means add RE images to VE, such as add texture mapping video on virtual objects. This term can increase virtual object's reality degree, decrease virtual object and real object's differences. But VE is entire virtual. In AR real object and virtual object and user environment must be seamlessly integrated together [17], [18].

\section{Android Platform}

Android is a software stack for mobile devices which means a reference to a set of system programs or a set of application programs that form a complete system. This software platform provides a foundation for applications just like a real working platform.

Android is a software platform and operating system for mobile devices, based on the Linux kernel, and developed by Google and later the Open Handset Alliance. It allows developers to write managed code in the Java language, controlling the device via Google-developed Java libraries. Applications written in $\mathrm{C}$ and other languages can be compiled to ARM native code and run, but this development path is not officially supported by Google.

Android is a free, open source and fully customizable mobile platform based on the Linux kernel. Android offers a full vertical software stack: an operating system, middleware and key applications. It also contains a rich set of APIs that allows third-party developers to develop great applications.

\section{E. Learning Theories}

In psychology and education, learning theories are attempts to describe how people and animals learn; thereby helping us understand the inherently complex process of learning. There are three main categories (philosophical frameworks) under which learning theories fall: behaviourism, cognitivism, and constructivism [19], [20].

Our core intension is to use the cognitive learning theory to make the mobile translating application. Cognitive theory is a learning theory of psychology that attempts to explain human behaviour by understanding the thought processes. The assumption is that humans are logical beings that make the choices that make the most sense to them. Information processing is a commonly used description of the mental process, comparing the human mind to a computer [19]-[21].

\section{RESEARCH METHODOLOGY}

\section{A. Objectives of the Research}

The purpose of the project is to improve and enhance communication between people who are speaking different languages that is, to try get rid of the language barrier among people who speak different languages anywhere to any environment whenever desired (ubiquitous). The following are the objectives of the research:

1) To develop the Mobile Augmented Reality application that will translate/decode Bahasa language into English language ubiquitous.

2) To study the suitable method of designing and developing a real time augmented reality translator in the mobile phone

3) To observe, simulate and test a mobile application system that will translate information in real time at the same time using usability and functionality testing system.

\section{B. Development Methodology}

Many researchers use different forms of methods to develop mobile application. This project development methodology is based on mobile Augmented Reality application that supports real time translation of words ubiquitous. However, Visual Cognitive-Simulative Software Development Life Cycle method will be implemented on the development of this application. Fig. 2 shows the Visual Cognitive-Simulative Software Development Life Cycle method which comprises of 5 different stages namely analysis, system design, system implementation and testing (Fig. 2).

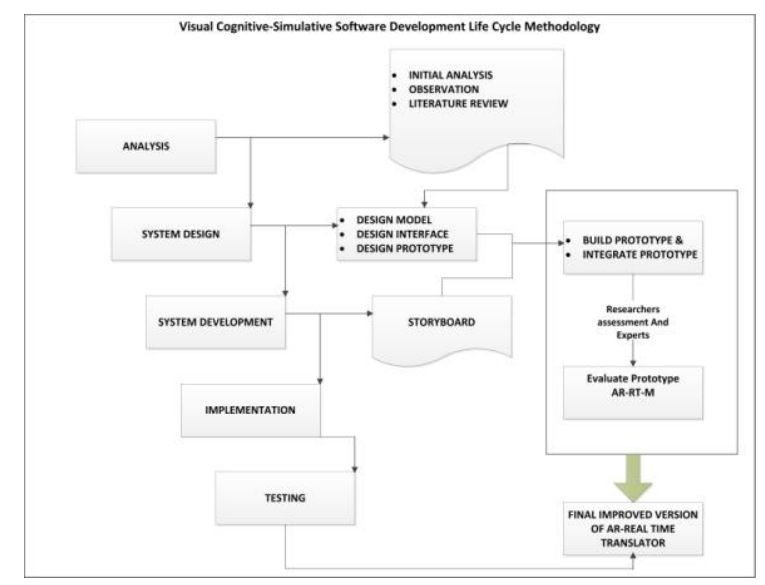

Fig. 2. Visual cognitive -simulative software development life cycle methodology

\section{Proposed Techniques}

The mobile device is the most ubiquitous device and a part of most people's everyday life. Hence, as mobile devices 
become more advanced, the development of 3D information systems for mobile users is a growing research area. Being mobile means that the context and the information changed, and computation can follow the user. We would like to exploit the features of a mobile device while trying to work around its limitations in order to study its potential to enhance the experience of the users. We have chosen to work with smartphones since they have the capability of rendering and displaying graphics such as video and 3D animations. They are connected to data enabled networks such as GPRS and UMTS and many of them now feature built in cameras [21]-[23]. The proposed application is to perform by capturing the words in Bahasa language and translates them to English language everywhere, anywhere whenever desired. On the other hand a directory/dictionary like Wikipedia, oxford dictionary and so on, will be embedded on to the application so that after the application has finished translating the directory/dictionary will generate meaning of that word. Fig. 3 below shows the design model which is a representation of the proposed application in chart form with the resources, objectives, theory, strategy and approach and interactivity clearly highlighted on the chart.

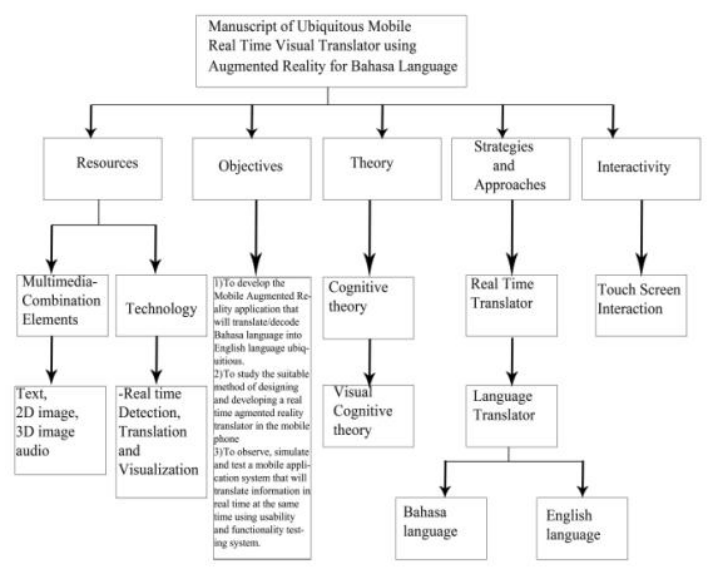

Fig. 3. Design model.

\section{Prototype}

The above Fig. 3 show the prototype or model of the proposed application. The user will have to have a mobile phone with a compatible application. By pointing a mobile camera at the any word and generate the translation on the application, thereby translating the word to another language at the same time getting the meaning of that word. The above Fig. 4 on its left shows a mobile phone pointing or displaying the word "sekolah" in Bahasa language (word before translation) and, on the other side it shows the mobile phone point at the word "school" and some explanation below it (it shows the everything after translation).
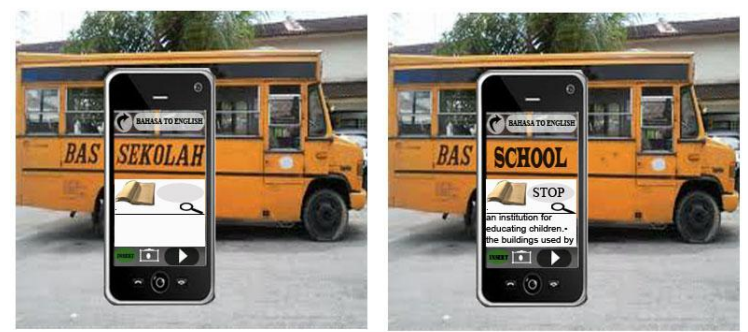

Fig. 4. Example of real time translator display on mobile phones.

\section{DISCUSSION AND FUTURE WORK}

The works of Mobile Augmented reality have been carried out since back in the days until today even more application are still made. So far the proposed application has been done to a certain limit because of certain reason like less time to do or implement other feature. This application we proposed translate only word by word so for future enhancement the application maybe improved to translate sentences, paragraphs and son on. In future we can also work on adding a feature that will allow images to be translated to words and vice versa or displaying $3 \mathrm{D}$ images that relate to each word translated to improve understandability.

\section{CONCLUSION}

Augmented reality technology is gradually growing and is dynamically changing a lot of things happening around the world. Moreover augmented reality is an effective and more efficient way for people to learn. The learning theory allows the users to attempt to explain human behavior by understanding the thought processes. The assumption is that humans are logical beings that make the choices that make the most sense to them. The augmented reality technology is now very easy to introduce using the mobile phones since they are ubiquitous. This is due to the advancement in the mobile phone technology, thus the platforms available as well as the interactivity techniques. In this research the mobile phone is used to simulate the events in the virtual world in to the real world. The end users will be able to see the translated word popping through the mobile phone screen with the Wikipedia link related to the word being translated. The system will be developed using the Visual Cognitive Simulative Software Development Life Cycle Methodology for Augmented Reality (V-CSSDLC-AR) for mobile based augmented visual translator.

\section{ACKNOWLEDGEMENT}

The special thank goes to our helpful advisors Dr. Arash Habibi Lashkari as research advisor of Postgraduate Center of study (PGC), for his supervision and guidance in the progression of our dissertation and project.

\section{REFERENCES}

[1] T. H. Höllerer, "User Interfaces for Mobile Augmented Reality Systems," Mobile Augmented Reality Systems, 2004, pp. 26-29.

[2] T. H. Höllerer and S. K. Feiner, "Mobile Augmented Reality," Defination, 2004, pp. 1-2.

[3] A. Henrysson and M. Ollila, "Ubiquitous Mobile Augmented Reality," UMAR Ubiquitous Mobile Augmented Reality, 2004, pp. 1-5.

[4] H. Benko, E. W. Ishak, and S. Feiner, "Collaborative mixed reality visualization of an archaeological excavation," in Prco. ISMAR, pp. 132-140, 2004.

[5] J. Newman, M. Wagner, M. Bauer, A. MacWilliams, T. Pintaric, D Beyer, D. Pustka, F. Strasser, D. Schmalstieg, and G. Klinker, "Ubiquitous tracking for augmented reality," in Prco. ISMAR, pp. 192-201, 2004.

[6] M. Möhring, C. Lessig, and O. Bimber, "Video See-Through AR on Consumer Cell Phones," in Proc. the 3th IEEE/ACM international Symposium on Mixed and Augmented Reality, 2004, pp. 252-253.

[7] A. Backman, "Augmented Reality," 2005, vol. 11, no. 1, pp. 1-24

[8] O. Bimber, G. Wetzstein, A. Emmerling, and C. Nitschke, "Enabling view-dependent stereoscopic projection in real environments," in Proc. ISMAR, pp. 14-23, 2005. 
[9] A. State, K. Keller, and H. Fuchs, "Simulation-based design and rapid prototyping of a parallax-free, orthoscopic video see-through headmounted display," in Proc. ISMAR, pp. 28-31, 2005.

[10] A. Henrysson, M. Billinghurst, and M. Ollila, "Face to Face Collaborative AR on Mobile Phones," in Proc. the 4th IEEE/ACM International Symposium on Mixed and Augmented Reality, 2005, pp. 80-89.

[11] H. Wuest, F. Vial, and D. Stricker, "Adaptive line tracking with multiple hypotheses for augmented reality," in Proc. ISMAR, pp. 62-69, 2005.

[12] S. Irawati, S. Green, M. Billinghurst, A. Duenser, and K. Heedong, "Move the couch where? developing an augmented reality multimodal interface," in Proc. ISMAR, pp. 183-186, 2006.

[13] G. Reitmayr and T. Drummond, "Going Out: Robust Model-based Tracking for Outdoor Augmented Reality," in Proc. 5th IEEE and ACM International Symposium on Mixed and Augmented Reality, 2006 pp. 109-118.

[14] P. Fua and V. Lepetit, "Vision based 3D tracking and pose estimation for mixed reality," in M. Haller, M. Billinghurst, and B. H. Thomas Eds, Emerging Technologies of Augmented Reality Interfaces and Design, pp. 43-63, Idea Group, Hershey, 2007.

[15] G. Klein and D. Murray, "Parallel tracking and mapping for small AR workspaces," in Proc. 6th IEEE and ACM International Symposium on Mixed and Augmented Reality, pp. 225-234, 2007

[16] S. DiVerdi and T. Höllerer, "GroundCam: A Tracking Modality for Mobile Mixed Reality," in Proc. the 9th international Conference on Virtual Reality, 2007, pp. 75-82.

[17] S. White, L. Lister, and S. Feiner, "Visual hints for tangible gestures in augmented reality," in Proc. ISMAR, pp. 47-50, 2007.

[18] S. White, L. Lister, and S. Feiner, "Visual hints for tangible gestures in augmented reality," in Proc. ISMAR, pp. 47-50, 2007.

[19] S. Walker. (2008). Learning Theories Self-growth. [Online]. Available: http://www.selfgrowth.com/articles/definition_learning theories.html

[20] B. Speckmann, "The Android mobile platform," What is Android, 2008 pp. 11-14.

[21] E. N. G. Wen, B. Parhizkar, L. C. H. Ping, and A. H. Lashkari, "Augmented reality for museum artifacts visualization," Augmented Reality, 2011, vol. 8, no. 4, pp. 4.

[22] M. Jantscher, M. Talhaoui, D. De Vos, I. Cunha, A. Roszcyk, and M. Datsyuk, "Android," What is Android? pp. 2-4, 2011

[23] L. Fritscher. Cognitive Theory. (April 02, 2011). About.com Guide Updated. [Online]. Available: http://www.phobias.about.com/od/glossary/g/cognitivethedef.htm

Behrang Parhizkar obtained his bachelor degree in Multimedia Technology Management from Multimedia University, Malaysia, followed by master degree of Information Technology \& Multimedia System, faculty of Information science and technology, University of Kebangsaan Malaysia (UKM), Bangi, Malaysia. He is a member of Visual Informatics research group. He is a principal lecturer and director of computer research center in Limkokwing University of Creative technology, Malaysia. He won some International awards such as Itex Bronz Medal of Innovation, Malaysia, Gold Medal of Invention, South Korea, and World Intellectual Property Organization (WIPO) Award for Voice Recognition Browser and Marking System. He is interested to research in Augmented Reality, Mobile Technology, Artificial Intelligent, Voice Recognition and Image Processing. He also works on Augmented Reality Technology, as a research assistant

Keamogetswe Oteng obtained his bachelor degree in Software Engineering with Multimedia from Limkokwing University of Creative technology, Malaysia. He was one of the international researchers in Computer research center in Limkokwing University of Creative technology.

One Ndaba obtained her bachelor degree in Software Engineering with Multimedia from Limkokwing University of Creative technology, Malaysia. She was one of the international researchers in Computer research center in Limkokwing University of Creative technology.

Arash Habibi Lashakri obtained his bachelor degree in software engineering 0n from IAU, Gilan, Iran, followed by master degree of computer system \& technology, faculty of computer science and information technology (FSKTM), University of Malaya (UM), Kuala Lumpur, Malaysia $\mathrm{He}$ is senior lecturer and research advisor of postgraduate center of study (PGC) in Limkokwing University of Creative technology, Malaysia. He is a reviewer of a few International journal of computer science and information security and Technical Committee member of some IEEE conferences based on computer science and information technology. He is interested to research in network security, cryptography, Augmented Reality, and especially graphical user authentication (GUA) subjects. He also works on wireles security, mobile communication, and optical network bandwidth allocation systems as a research assistant

Zahra Mohana Gebril obtained her bachelor degree in Information Technology majoring in Multimedia Systems from Multimedia University, Malaysia, followed by master degree of Multimedia (E-learning Technologies), faculty of Creative Multimedia, Multimedia University, Malaysia. She is a member of Visual Informatics research group. She is a Multimedia Lecturer in Limkokwing University of Creative technology, Malaysia. She is interested to research in affective computing, Human computer Interaction, Mobile Technology, Multimedia systems and Image Processing. 